\title{
Correction to: Uniqueness for nonlinear Fokker-Planck equations and weak uniqueness for McKean-Vlasov SDEs
}

\author{
Viorel Barbu ${ }^{1} \cdot$ Michael Röckner ${ }^{2,3}$
}

Received: 22 October 2021 / Revised: 22 October 2021 / Accepted: 23 October 2021 /

Published online: 17 November 2021

(c) Springer Science+Business Media, LLC, part of Springer Nature 2021

\begin{abstract}
A gap in the proof of the main result in the above paper is filled in this work.

Keywords Fokker-Planck equation · Mild solution · Distributional solution

Mathematics Subject Classification $60 \mathrm{H} 30 \cdot 60 \mathrm{H} 10 \cdot 60 \mathrm{G} 46 \cdot 35 \mathrm{C} 99$

\section{Correction to: Stoch PDE: Anal Comp https://doi.org/10.1007/s40072-020-00181-8}

\section{Introduction}

Due to a misprint in [1], it was overlooked that an argument was missing in the proof of its Theorems 2.1 and 3.1. In the next section, we fill this gap in the case of Theorem 2.1. For Theorem 3.1, this can be done analogously. Let us, briefly, recall the situation and conditions which in regard to [1] are weakened, since we only deal with the two mentioned theorems.
\end{abstract}

The original article can be found online at https://doi.org/10.1007/s40072-020-00181-8.

$凶$ Viorel Barbu

vbarbu41@gmail.com

Michael Röckner

roeckner@math.uni-bielefeld.de

1 Octav Mayer Institute of Mathematics of the Romanian Academy, Iaşi, Romania

2 Fakultät für Mathematik, Universität Bielefeld, 33501 Bielefeld, Germany

3 Academy of Mathematics and Systems Science, CAS, Beijing, China 
Consider the equation

$$
\begin{aligned}
& u_{t}-\Delta \beta(u)+\operatorname{div}(b(x, u) u)=0 \text { in } \mathcal{D}^{\prime}\left((0, \infty) \times \mathbb{R}^{d}\right) \\
& u(0, x)=u_{0}(x), x \in \mathbb{R}^{d}, 0<T<\infty
\end{aligned}
$$

\section{Assumptions}

(i) $\beta \in C^{1}(\mathbb{R}), \beta(0)=0$,

$$
0<\gamma_{0} \leq \beta^{\prime}(r), \forall r \in \mathbb{R}
$$

(ii) $b \in C_{b}\left(\mathbb{R}^{d+1} ; \mathbb{R}^{d}\right), b(x, \cdot) \in C^{1}, \forall x \in \mathbb{R}^{d}$, such that

$$
\sup \left\{\left|b_{r}(x, r)\right| ; x \in \mathbb{R}^{d},|r| \leq M\right\} \leq C_{M}, \forall M
$$

Definition. $u:[0, T) \rightarrow L^{1}\left(\mathbb{R}^{d}\right)$ is said to be a distributional solution to (1) if $[0, T] \in t \rightarrow u(t, . x) d x$ is narrowly continuous and

$$
\begin{aligned}
& \int_{0}^{T} \int_{\mathbb{R}^{d}}\left(u \varphi_{t}+\beta(u) \Delta \varphi+b(x, u) u \cdot \nabla_{x} \varphi\right) d t d x+\int_{\mathbb{R}^{d}} u_{0}(x) \varphi(0, x) d x=0, \\
& \quad \forall \varphi \in C_{0}^{\infty}\left([0, T) \times \mathbb{R}^{d}\right) .
\end{aligned}
$$

Theorem 1 For each $u_{0} \in L^{1} \cap L^{\infty}$, there is at most one distributional solution

$$
u \in L^{1}\left(0, T ; L^{1}\right) \cap L^{\infty}\left((0, T) \times \mathbb{R}^{d}\right)
$$

to $(1)$.

Before we prove this theorem, let us point out the misprint in [1].

In the proof of Theorem 2.1, on the left hand side of the equality following formula (2.2), $y(t)$ in the right slot of $\langle\cdot, \cdot\rangle_{2}$ should have been $u(t)$ and thus the equality should be

$$
\left\langle\frac{d y}{d t}(t), u(t)\right\rangle_{2}=\frac{1}{2} \frac{d}{d t}|u(t)|_{-1}^{2} .
$$

As a consequence, in the rest of the proof $|u(t)|_{-2}^{2}$ should be replaced by $|u(t)|_{-1}^{2}$ to arrive at the final conclusion

$$
\frac{d}{d t}|u(t)|_{-1}^{2} \leq C|u(t)|_{-1}^{2} \text {, a.e. } t \in[0, T]
$$

which implies $u \equiv 0$ as before. Here, $u=u_{1}-u_{2}$, where $u_{1}, u_{2}$ are two distributional solutions to (1) with initial condition $u_{0} \in L^{1} \cap L^{\infty}$. However, the argument to justify the above is that we have to prove that $u_{1}, u_{2}$ satisfy condition (6) in Section 2 below. 


\section{Proof of Theorem 1}

Assume first that, in addition,

$$
u \in L^{2}\left(0, T ; H^{1}\right) ; u \in W^{1,2}\left([0, T] ; H^{-1}\right)
$$

Then the uniqueness follows as in V. Barbu and M. Röckner [1].

To conclude the proof, we shall prove

Lemma 2 If $u$ is a distributional solution to (1) satisfying (5), then (6) holds.

Proof of Lemma $21^{\circ}$. We shall first assume that $u$ satisfies the stronger initial condition

$$
\text { essential } \lim _{t \rightarrow 0} \int_{\mathbb{R}^{d}}\left|u(t, x)-u_{0}(x)\right| d x=0 .
$$

Next, we consider the truncated function

$$
\beta_{M}(r)= \begin{cases}\beta(r) & \text { if }|r| \leq M \\ \beta(M)+\beta^{\prime}(M)(r-M) & \text { if } r>M \\ \beta(-M)+\beta^{\prime}(-M)(r+M) & \text { if } r<-M\end{cases}
$$

where $M=\|u\|_{\left.L^{\infty}(0, T) \times \mathbb{R}^{d}\right)}$.

We set

$$
f(t, x) \equiv b^{*}(x, u(t, x)), \quad f_{v}=f * \rho_{\nu}, \quad v>0,
$$

where $\rho_{v}$ is a standard mollifier on $\mathbb{R}^{d+1}$ and $b^{*}(x, u) \equiv b(x, u) u$.

We note that in equation (1) one can take $\beta \equiv \beta_{M}$ because $|u|_{\infty} \leq M$.

We consider the equations

$$
\begin{aligned}
& \tilde{u}_{t}-\Delta \beta_{M}(\widetilde{u})+\operatorname{div}(f)=0 \text { in }(0, T) \times \mathbb{R}^{d}, \\
& \widetilde{u}(0)=u_{0}, \\
& \left(\widetilde{u}_{v}\right)_{t}-\Delta \beta_{M}\left(\tilde{u}_{v}\right)+\operatorname{div}\left(f_{v}\right)=0 \text { in }(0, T) \times \mathbb{R}^{d}, \\
& \tilde{u}_{v}(0)=u_{0} .
\end{aligned}
$$

Clearly, (9) has a unique solution $\tilde{u}$ satisfying (6), i.e.,

$$
\tilde{u} \in L^{2}\left(0, T ; H^{1}\right), \tilde{u} \in W^{1,2}\left([0, T] ; H^{-1}\right),
$$

while $\tilde{u}_{v}$ is more regular, that is, additionally to (11) it satisfies

$$
\tilde{u}_{v} \in L^{\infty}\left((0, T) \times \mathbb{R}^{d}\right) \cap C^{1}\left([0, T] ; L^{1}\right),
$$


because the operator $u \rightarrow-\Delta \beta_{M}(u)$ is $m$-accretive in $L^{1}\left(\mathbb{R}^{d}\right)$ and $u_{0} \in\left(L^{1} \cap\right.$ $\left.L^{\infty}\right)\left(\mathbb{R}^{d}\right), \operatorname{div} f_{v} \in\left(L^{1} \cap L^{\infty}\right)\left((0, T) \times \mathbb{R}^{d}\right)$. It is also clear that

$$
\lim _{\nu \rightarrow 0} \tilde{u}_{v}=\tilde{u} \text { strongly in } L^{2}\left(0, T ; H^{-1}\right) .
$$

To prove Lemma 2, we are going to show that

$$
\tilde{u}=u \text {, a.e. in }(0, T) \times \mathbb{R}^{d} .
$$

To this end, we shall invoke an argument due to Brezis \& Crandall [2].

Namely, we subtract equations (1), (10) and get

$$
\begin{aligned}
& \left(\tilde{u}_{v}-u\right)_{t}-\Delta\left(\beta\left(\tilde{u}_{v}\right)-\beta(u)=-\operatorname{div}\left(f_{v}-f\right) \text { in } \mathcal{D}^{\prime}\left((0, T) \times \mathbb{R}^{d}\right),\right. \\
& \text { essential } \lim _{t \rightarrow 0} \int_{\mathbb{R}^{d}}\left|\tilde{u}_{v}(t, x)-u(t, x)\right| d x=0
\end{aligned}
$$

We set $z_{v}=\tilde{u}_{v}-u, h_{v}=\beta\left(\tilde{u}_{v}\right)-\beta(u)$ and get

$$
\begin{aligned}
& \left(z_{\nu}\right)_{t}-\Delta h_{v}=-\operatorname{div}\left(f_{v}-f\right) \text { in } \mathcal{D}^{\prime}\left((0, T) \times \mathbb{R}^{d}\right), \\
& \text { essential } \lim _{t \rightarrow 0} \int_{\mathbb{R}^{d}}\left|z_{\nu}(t, x)\right| d x=0 .
\end{aligned}
$$

Arguing as in [2] (proof of Proposition 1), we set

$$
g_{\varepsilon}^{\nu}(t)=\left(B_{\varepsilon}\left(z_{v}\right), z_{v}\right), B_{\varepsilon}=(\varepsilon I-\Delta)^{-1},
$$

where ( , ) denotes inner product in $L^{2}=L^{2}\left(\mathbb{R}^{d}\right)$, and get

$$
\left(g_{\varepsilon}^{v}(t)\right)_{t}=2\left(\varepsilon B_{\varepsilon}\left(h_{v}\right)-h_{v}, z_{v}(t)\right)+2\left(f_{v}-f, \nabla B_{\varepsilon}\left(z_{v}\right)\right)
$$

and, therefore (we note that $\left(h_{v}, z_{v}\right) \geq 0$ ),

$$
g_{\varepsilon}^{v}(t) \leq 2 \int_{0}^{t} \varepsilon\left(B_{\varepsilon}\left(h_{v}(s)\right), z_{v}(s)\right) d s+2 \int_{0}^{t}\left(\left(f_{v}-f\right)(s), \nabla B_{\varepsilon}\left(z_{v}(s)\right)\right) d s
$$

because, by (16), we have, since $z_{v} \in L^{\infty} \cap L^{1}$,

$$
g_{\varepsilon}^{v}\left(0^{+}\right)=\text {essential } \lim _{t \rightarrow 0}\left(B_{\varepsilon}\left(z_{v}(t), z_{v}(t)\right)=0 .\right.
$$

Recalling that, for a.e. $s \in[0, T]$,

$$
\varepsilon B_{\varepsilon}\left(z_{v}(s)\right)-\Delta B_{\varepsilon}\left(z_{v}(s)\right)=z_{v}(s),
$$

we get

$$
g_{\varepsilon}^{\nu}(s)=\left(B_{\varepsilon}\left(z_{\nu}(s)\right), z_{\nu}(s)\right)=\varepsilon\left|B_{\varepsilon}\left(z_{v}(s)\right)\right|_{L^{2}}^{2}+\left|\nabla B_{\varepsilon}\left(z_{v}(s)\right)\right|_{L^{2}}^{2}
$$


and so, by (17), we have

$$
g_{\varepsilon}^{v}(t) \leq 2 \int_{0}^{t} \varepsilon\left(B_{\varepsilon} h_{v}(s), z_{v}(s)\right) d s+\int_{0}^{t}\left|\left(f_{v}-f\right)(s)\right|_{L^{2}}^{2} d s+\int_{0}^{t} g_{\varepsilon}^{v}(s) d s .
$$

Hence, by Gronwall's inequality,

$$
g_{\varepsilon}^{v}(t) \leq \int_{0}^{t}\left(2 \varepsilon\left(B_{\varepsilon}\left(h_{v}(s)\right), z_{v}(s)\right)+\left|\left(f_{v}-f\right)(s)\right|_{L^{2}}^{2}\right) e^{t-s} d s .
$$

On the other hand, by Lemma 1 in [2] we have for a.e. $s \in[0, T]$ and also in $L^{1}(0, T)$

$$
\lim _{\varepsilon \rightarrow 0}\left(\varepsilon B_{\varepsilon}\left(h_{v}(s)\right), z_{v}(s)\right)=0 .
$$

By (20), this yields

$$
\left(G * z_{\nu}(t), z_{v}(t)\right) \leq \int_{0}^{t}\left|\left(f_{v}-f\right)(s)\right|_{L^{2}}^{2} e^{t-s} d s
$$

since

$$
\lim _{\varepsilon \rightarrow 0} g_{\varepsilon}^{\nu}(t)=\left((-\Delta)^{-1} z_{v}(t), z_{v}(t)\right)=\left(G * z_{v}(t), z_{v}(t)\right)
$$

where $G$ is the classical Newtonian Green function. We note that the latter inner product is well defined, since $z_{v} \in \bigcap_{p \in[1, \infty]} L^{p}$ and hence $G * z_{v} \in L^{\frac{2 d}{d-2}}$. Letting $v \rightarrow 0$ in (22), we find

$$
\lim _{\nu \rightarrow 0}\left(G * z_{v}(t), z_{v}(t)\right)=0
$$

In particular, $z_{v}(t) \underset{v \rightarrow 0}{\longrightarrow} 0$ in $H^{-1}$. Since $z_{v} \underset{v \rightarrow 0}{\longrightarrow} \tilde{u}-u$ in $L^{2}\left(0, T ; L^{2}\right)$ by (13), we have along a subsequence $z_{v}(t) \underset{v \rightarrow 0}{\longrightarrow}(\tilde{u}-u)(t)$ in $L^{2}$ for a.e. $t \in[0, T]$, hence (14) follows.

$2^{\circ}$. The proof without assuming (7). It follows by the argument described under (1.12) in [2] that (4) indeed implies (18). So, assumption (7) can be dropped.

This completes the proof. 


\section{References}

1. Barbu, V., Röckner, M.: Uniqueness for nonlinear Fokker-Planck equations and weak uniqueness for McKean-Vlasov SDEs. Stoch. PDE: Anal. Comp. (2020). https://doi.org/10.1007/s40072-020-001818

2. Brezis, H., Crandall, M.G.: Uniqueness of solutions of the initial-value problem for $u_{t}-\Delta \beta(u)=0$. J. Math. Pures et Appl. 58, 153-163 (1979)

Publisher's Note Springer Nature remains neutral with regard to jurisdictional claims in published maps and institutional affiliations. 